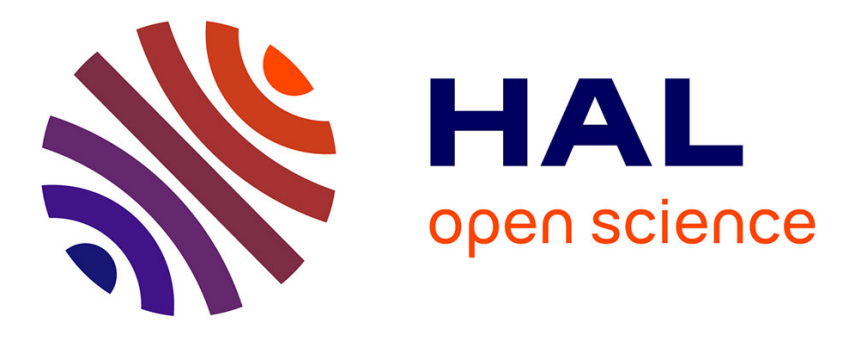

\title{
Radar Cross-Section Pattern Measurements in a Mode-Stirred Reverberation Chamber: Theory and Experiments
}

Ariston Reis, Francois Sarrazin, Elodie Richalot, Stephane Meric, Jerome Sol, Philippe Pouliguen, Philippe Besnier

\section{To cite this version:}

Ariston Reis, Francois Sarrazin, Elodie Richalot, Stephane Meric, Jerome Sol, et al.. Radar Cross-Section Pattern Measurements in a Mode-Stirred Reverberation Chamber: Theory and Experiments. IEEE Transactions on Antennas and Propagation, 2021, 69 (9), pp.5942 - 5952. 10.1109/TAP.2021.3060581 . hal-03157142

\section{HAL Id: hal-03157142 \\ https://hal.science/hal-03157142}

Submitted on 2 Mar 2021

HAL is a multi-disciplinary open access archive for the deposit and dissemination of scientific research documents, whether they are published or not. The documents may come from teaching and research institutions in France or abroad, or from public or private research centers.
L'archive ouverte pluridisciplinaire HAL, est destinée au dépôt et à la diffusion de documents scientifiques de niveau recherche, publiés ou non, émanant des établissements d'enseignement et de recherche français ou étrangers, des laboratoires publics ou privés. 


\title{
Radar Cross-Section Pattern Measurements in a Mode-Stirred Reverberation Chamber: Theory and Experiments
}

\author{
Ariston Reis, François Sarrazin, Member, IEEE, Elodie Richalot, Member, IEEE, Stéphane Méric, Member, IEEE,
} Jérôme Sol, Philippe Pouliguen, and Philippe Besnier, Senior Member, IEEE

\begin{abstract}
This paper deals with the ability to perform radar cross-section (RCS) pattern measurements within reverberation chambers (RCs). The characterization principle is based on the estimation of the back-scattered field from the target at a farfield distance and takes advantage of the diffuse field within the RC. A frequency sweep of scattering parameters is achieved in presence and absence of the target installed on a rotating mast and the pattern is retrieved from these measurements. This paper explains the underlying theory and discusses the impact of several parameters on the performances. This includes the effect of the stirrer rotation which enables to enhance the dynamic range of the measurements. Experiments in two different RCs show the relevance of the proposed approach for RCS extraction and confirm the theoretical analysis.
\end{abstract}

Index Terms-Radar cross section, reverberation chamber, measurement.

\section{INTRODUCTION}

$\mathbf{E}$ LECTROMAGNETIC reverberation chambers (RCs) have been studied for decades for electromagnetic compatibility applications following early works in [1]. Since then, their range of application has been extended to the field of antennas and propagation. Antenna characterization in an RC was addressed as early as in 2001 [2]. More recently, a few papers discussed the ability of RCs to perform radar crosssection (RCS) measurements. It was initially proposed in [3] to use an RC to estimate the average scattering cross-section of an object, i. e. its cross-section integrated over all angles of incidence and both polarizations due to field diffuseness within the RC. Indeed, displacing the target in a statistical isotropic and homogeneous electromagnetic field allows to retrieve its average scattering cross-section. Beyond this global scattering indicator, the estimation in an RC of the RCS pattern of a target remains a challenge. Although not targeted for this application, the time reversal electromagnetic chamber (TREC) [4] may be of help to generate the target illumination through the electronic control of wave-front angle of incidence and polarization. However, the control of the TREC requires a sophisticated calibration process which, moreover, may be influenced by the presence of the target.

A. Reis, F. Sarrazin and E. Richalot are with ESYCOM lab, Univ Gustave Eiffel, CNRS UMR 9007, F-77454 Marne-la-Vallée.

P. Pouliguen is with the Defence Innovation Agency, Ministry of the Armed Forces.

S. Méric, J. Sol and P. Besnier are with Univ Rennes, INSA Rennes, CNRS, IETR-UMR 6164, F-35000 Rennes, France.

Manuscript received March 22, 2020; revised September 1, 2020.

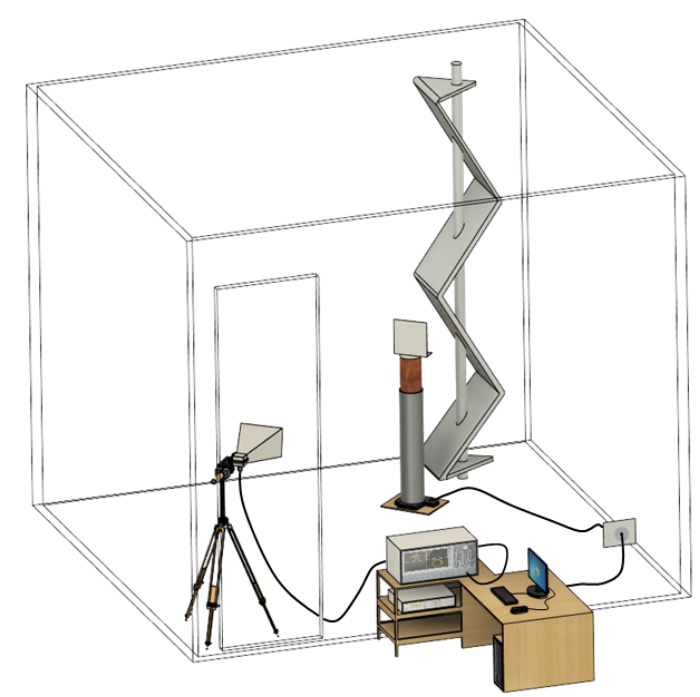

Fig. 1. Schematic of the RCS measurement setup within RC.

Another family of solutions was investigated, based on the distinction between direct and diffuse paths between a transmitting (Tx) antenna and an object, the latter being possibly a receiving ( $\mathrm{Rx})$ antenna [5]. In this latter case, the extraction of the ballistic (direct path) field component among the total back-scattered field has been used for antenna directivity characterization from measurements in RC. Indeed, two coupled antennas in an RC generate a Rice distributed field resulting from the superposition of a diffuse field and a ballistic wave between both antennas. The line-ofsight unstirred field component between both antennas can be evaluated through the $K$-factor estimation of this Rice probability density function describing the RC propagation channel; this process requires both mechanical and frequency stirring for an acceptable estimation. The obtained $K$-factor is proportional to antennas directivity, and the rotation of one antenna then leads to an estimation of its directivity pattern. Another approach has been proposed to distinguish the line-of-sight component between two antennas from all other multipath field components within an RC and to extract antenna radiation pattern [6]. It consists in moving, step by step, the measurement antenna toward the antenna under test, and exploiting the real-time Doppler effect to identify the ballistic wave associated to the maximum frequency shift. On 
the contrary, the stirred (non-line-of-sight) field components are used in [7] to determine the coefficients of the antenna radiation pattern decomposition into spherical harmonics; this approach is based on the calculus of self-correlation coefficients when rotating the antenna under test.Another approach consists in recording the response of the test environment, that can be an RC, and selecting the line-of-sight path contribution through a time-gating post-process. Such approach has been proposed to measure the radiation pattern of an antenna [8] as well as the RCS of a target [9]. However such an approach requires a new definition of the gating internals for each target.

Some co-authors of the present paper have proposed a novel technique providing an estimation of RCS patterns based on frequency stirring and extraction of the target's signature from complex-valued scattering parameters of a single antenna pointing towards the target [10]. This technique takes advantage of the diffuse field properties within the RC. As these preliminary works did not investigate neither the effect of frequency step and bandwidth nor any mechanical stirring, the mechanical stirrer remaining in a fixed position, the present paper aims at three main goals. First, it provides a theory update of the RCS measurement technique by taking into account the stirrer effect. Second, a statistical model based on this theory provides some important insights into the choice of frequency step and bandwidth in relationship with the coherence bandwidth (or $Q$-factor) within the chamber. Third, it introduces the mechanical stirring as a tool to enhance the accuracy of the RCS estimation for low signal-to-noise ratio (SNR) configurations.

The paper is organized as follows. Section II presents the theory of the RCS measurement within RC. A numerical analysis is performed in section III in order to highlight the key parameters to enhance the RCS extraction accuracy. After a brief presentation of the considered targets and measurement platforms (an anechoic chamber (AC) and two RCs) in section $\mathrm{IV}$, the introduced technique is applied to measure the RCS pattern of a metallic plate in section V. Finally, the method is validated using a second target in section VI. Finally, we draw some conclusions in section VII.

\section{THEORY OF RCS MEASUREMENTS IN RC}

This theory deals with mono-static RCS measurement in RC. It follows a former presentation in [10], [11] with some highlighted changes.

\section{A. Measurement set-up}

The measurement set-up consists of an horn antenna placed in an arbitrary location within the $\mathrm{RC}$ working volume and pointing towards the target (Fig. 1). The latter is installed on a rotating mast at the same height as the antenna; measurements are thus performed in the azimuthal plane for this configuration. The transmitting / receiving antenna is connected to a port of a vector network analyzer (VNA). The electromagnetic field illuminating the target differs from the free space configuration as the impinging wave from the antenna reaching the target at early time is followed by the contribution of wall reflections acting as secondary sources. However, the distance $R$ between the target and the antenna needs to satisfy the far-field condition in free space that is a distance larger than the Fraunhofer distance $2 D^{2} / \lambda$ where $D$ is the largest dimension of the target and $\lambda$ the minimum considered wavelength. In principle, this far-field condition should be extended to twice this distance. However, we stick the classical Fraunhofer distance due to our practical limitations. It permits to assume a quasi-plane ballistic wave at the target position. The antenna to target axis is arbitrary with regard to the chamber geometry and the stirrer location.

\section{B. Backscattered field in an empty RC (without target)}

The VNA generates a continuous wave (CW) signal of frequency $f_{0}$ (angular frequency $\omega_{0}$ ) higher than the threshold frequency, also referred as the Lowest Usable Frequency (LUF) [12]. Indeed, as deviations from the expected diffuse field are experimentally observed close to the LUF [13], our measurements will be conducted well above this limit. The scattering parameter measured by the VNA (once calibrated at the antenna's connector level) is composed of the intrinsic reflection coefficient of the antenna (measured in free space) in addition to the RC backscattered contribution [14]. The reflection coefficient measured in the empty cavity $S\left(f_{0}, \alpha_{\mathrm{st}}\right)$ at the frequency $f_{0}$ and for the stirrer angular position $\alpha_{\text {st }}$ can be expressed as:

$$
\begin{aligned}
& S\left(f_{0}, \alpha_{\mathrm{st}}\right)=S_{\mathrm{FS}}\left(f_{0}\right) \\
& \quad+\left(1-\left|S_{\mathrm{FS}}\left(f_{0}\right)\right|^{2}\right) \eta_{\mathrm{ant}}\left[H\left(f_{0}, \alpha_{\mathrm{st}}\right)+h_{\mathrm{s}}\left(f_{0}, \alpha_{\mathrm{st}}\right)\right]
\end{aligned}
$$

where $S_{\mathrm{FS}}$ stands for the antenna reflection coefficient in free space, $\eta_{\text {ant }}$ represents the antenna radiation efficiency, $H\left(f_{0}, \alpha_{\text {st }}\right)$ is a complex-valued transfer function describing the backscattered signal towards the antenna associated to the diffuse field (related to multiple reflections and diffractions within the cavity), and $h_{\mathrm{s}}\left(f_{0}, \alpha_{\mathrm{st}}\right)$ accounts for line-of-sight or specular reflections from the $\mathrm{RC}$ walls and mechanical stirrer towards the antenna. Both transfer functions depend on the stirrer angular position $\alpha_{\mathrm{st}}$, as shown experimentally in [15]. It has to be noticed that $h_{\mathrm{s}}\left(f_{0}, \alpha_{\mathrm{st}}\right)$ was not introduced in [10]. Assuming a perfectly diffuse field, both real and imaginary parts of $H\left(f_{0}, \alpha_{\mathrm{st}}\right)$ are described by random variables following a centred Gaussian distribution.

\section{Backscattered field from the target}

The addition of a target on the mast yields a modification of the previous equation according to two main hypotheses. We assume that the target is small enough to provide a small perturbation of the field. In other words, the addition of the target is dealt with through the Born approximation [16], stating that the diffuse field is only perturbed by the interaction between the target and the antenna. As a result, the previous equation is transformed into the following one: 


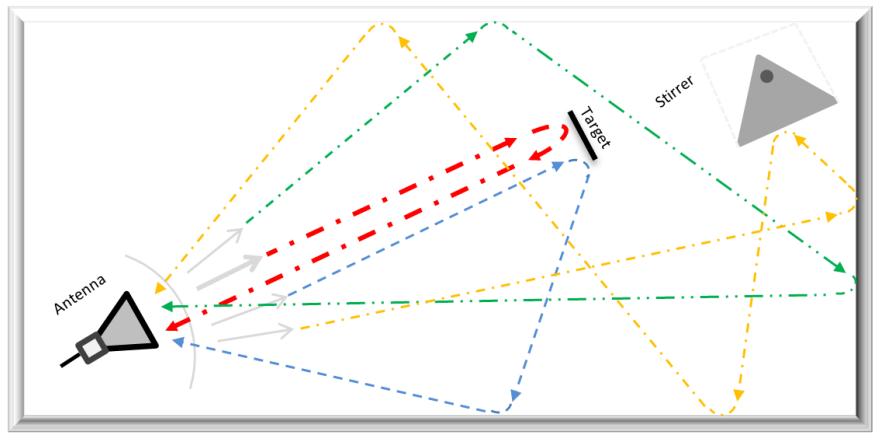

Fig. 2. Different possible paths within the $\mathrm{RC}$ in the presence of the target. In red: the specular reflection from the target, green: specular reflection from the RC, orange: multiple reflection and diffraction interacting with the mode stirrer, blue: specular reflections between the antenna, the target and the cavity walls (neglected).

$$
\begin{aligned}
& S_{\mathrm{T}}\left(f_{0}, \alpha_{\mathrm{st}}, \theta_{\mathrm{T}}\right)=S_{\mathrm{FS}}\left(f_{0}\right)+C\left(f_{0}\right) \sqrt{\sigma_{\mathrm{T}}\left(f_{0}, \theta_{\mathrm{T}}\right)} \\
& +\left(1-\left|S_{\mathrm{FS}}\left(f_{0}\right)\right|^{2}\right) \eta_{\mathrm{ant}}\left[H_{\mathrm{T}}\left(f_{0}, \alpha_{\mathrm{st}}, \theta_{\mathrm{T}}\right)+h_{\mathrm{s}}\left(f_{0}, \alpha_{\mathrm{st}}\right)\right]
\end{aligned}
$$

The additional term $C\left(f_{0}\right) \sqrt{\sigma_{\mathrm{T}}\left(f_{0}, \theta_{\mathrm{T}}\right)}$ corresponds to the ballistic wave backscattered by the target (at the orientation $\theta_{\mathrm{T}}$ ) towards the antenna (red path in Fig. 2). The complexvalued function $C\left(f_{0}\right)$ describes, at the frequency $f_{0}$, the wave propagation from antenna to target then from target to antenna. Specular reflections between the target, the cavity walls and the antenna are neglected (blue paths in Fig. 2), although they may have a significant amplitude for very specific target orientations. Finally $\sigma_{\mathrm{T}}\left(f_{0}, \theta_{\mathrm{T}}\right)$ is the RCS of the target at the same frequency and at the orientation $\theta_{\mathrm{T}}$. Though we considered the Born approximation, the diffuse field transfer function is not strictly equal to the one of the empty chamber, as a slight modification occurs due to the interaction of diffuse field with the target; however its statistics remain the same. The new transfer function is denoted $H_{\mathrm{T}}\left(f_{0}, \alpha_{\mathrm{st}}, \theta_{\mathrm{T}}\right)$. Unlike the initial theory in [10], it is explicit that the target is added in the chamber whereas the stirrer remains at position $\alpha_{\mathrm{st}}$. We therefore assume that the line-of-sight or specular reflection from the stirrer and the cavity walls $h_{\mathrm{s}}\left(f_{0}, \alpha_{\mathrm{st}}\right)$ is not affected by the presence of the target ; it means that the possible masking effect of the target is neglected here. Assuming the target in the antenna far-field area, we deduce from the radar equation:

$$
\left|C\left(f_{0}\right)\right|=\frac{G_{\text {ant }}\left(f_{0}\right) \lambda_{0}}{(4 \pi)^{3 / 2} R^{2}}\left(1-\left|S_{\mathrm{FS}}\left(f_{0}\right)\right|^{2}\right)
$$

As expected, the quantity $\left|C\left(f_{0}\right)\right|$ is proportional to the antenna gain $G_{\text {ant }}\left(f_{0}\right)$ and evolves with the inverse of the square distance $R$ between the antenna and the target. As the target is assumed to be in the far-field of the horn-antenna, its contribution to the backscattered wave is seen from the antenna as coming from a punctual source. The far-field requirement is hardly fullfilled for large ratios of $D / \lambda_{0}$, where $D$ is the largest dimension of the target. However, it is not a specific limitation of RCs. This leads to the phase of $C$ (accounting for an arbitrary constant phase $\left.\phi_{0}\right)$ :

$$
C\left(f_{0}\right)=\left|C\left(f_{0}\right)\right| \exp \left(-\mathrm{j} 2 \pi f_{0} \frac{2 R}{c}\right) \exp \left(\mathrm{j} \phi_{0}\right)
$$

where $c$ is the speed of light.

\section{RCS equation of the target}

Computing the difference between the measured scattering parameters in both configurations (with and without the target) allows to retrieve the response of the target with an expression for its RCS $\sigma_{\mathrm{T}}$.

$$
\begin{aligned}
& S_{\mathrm{T}}\left(f_{0}, \alpha_{\mathrm{st}}, \theta_{\mathrm{T}}\right)-S\left(f_{0}, \alpha_{\mathrm{st}}\right)= \\
& \sqrt{\sigma_{\mathrm{T}}\left(f_{0}, \theta_{\mathrm{T}}\right)} \times\left|C\left(f_{0}\right)\right| \times \exp \left[-\mathrm{j}\left(2 \pi f_{0} \frac{2 R}{c}-\phi_{0}\right)\right] \\
& +\left(1-\left|S_{\mathrm{FS}}\left(f_{0}\right)\right|^{2}\right) \eta_{\mathrm{ant}}\left[H_{\mathrm{T}}\left(f_{0}, \alpha_{\mathrm{st}}, \theta_{\mathrm{T}}\right)-H\left(f_{0}, \alpha_{\mathrm{st}}\right)\right]
\end{aligned}
$$

The first term on the right hand side of (5) contains the magnitude of the backscattered signal from the target. The second term represents the difference of the diffuse transfer functions of the chamber in the two different states, with and without the target. It acts as an interfering signal with regard to the backscattered wave in the line-of-sight from the target. Interestingly, it is proportional to the difference of two random variables, $H_{\mathrm{T}}\left(f_{0}, \alpha_{\mathrm{st}}, \theta_{\mathrm{T}}\right)$ and $H\left(f_{0}, \alpha_{\mathrm{st}}\right)$, whose real and imaginary parts are distributed according to a centred Gaussian probability density function with equal variance. For clarity, (5) can be rewritten as

$$
\begin{aligned}
& S_{\mathrm{T}}\left(f_{0}, \alpha_{\mathrm{st}}, \theta_{\mathrm{T}}\right)-S\left(f_{0}, \alpha_{\mathrm{st}}\right)= \\
& A\left(f_{0}, \theta_{\mathrm{T}}\right) \times \exp \left[-\mathrm{j}\left(2 \pi \frac{f_{0}}{\delta f}-\phi_{0}\right)\right]+n\left(f_{0}, \alpha_{\mathrm{st}}, \theta_{\mathrm{T}}\right)
\end{aligned}
$$

with $A\left(f_{0}, \theta_{\mathrm{T}}\right)$ the magnitude of a sine wave signal, $\delta f=$ $\frac{c}{2 R}$, and $n\left(f_{0}, \alpha_{\mathrm{st}}, \theta_{\mathrm{T}}\right)$ a complex noise following a centered Gaussian distribution.

Moreover, if we assume constant values for $\sigma_{\mathrm{T}}, G_{\text {ant }}$ and $S_{\mathrm{FS}}$ over some frequency range around $f_{0}$, the exponential exponent indicates that the first term on the right hand side of (5) behaves as a sine wave signal versus frequency, with a periodicity (in the frequency space) $\delta f$. Consequently, the determination of $\sigma_{\mathrm{T}}\left(f_{0}, \theta_{\mathrm{T}}\right)$ necessitates the extraction around the frequency $f_{0}$ of the magnitude $A\left(f_{0}, \theta_{\mathrm{T}}\right)$ of this sine wave signal. To allow this magnitude extraction, a frequency band centred around $f_{0}$ is considered. Its bandwidth is denoted $\Delta f$ such as $\Delta f=N \times \delta f_{s}$, where $N$ is the odd number of frequency steps of small excursion $\delta f_{s}$. Finally, the RCS is estimated from the following expression:

$$
\left|\sigma_{\mathrm{T}}\left(f_{0}, \theta_{\mathrm{T}}\right)\right| \approx A\left(f_{0}, \theta_{\mathrm{T}}\right)^{2} \frac{(4 \pi)^{3} R^{4}}{\left(1-\left|S_{\mathrm{FS}}\left(f_{0}\right)\right|^{2}\right)^{2} G_{\mathrm{ant}}^{2}\left(f_{0}\right) \lambda_{0}^{2}}
$$




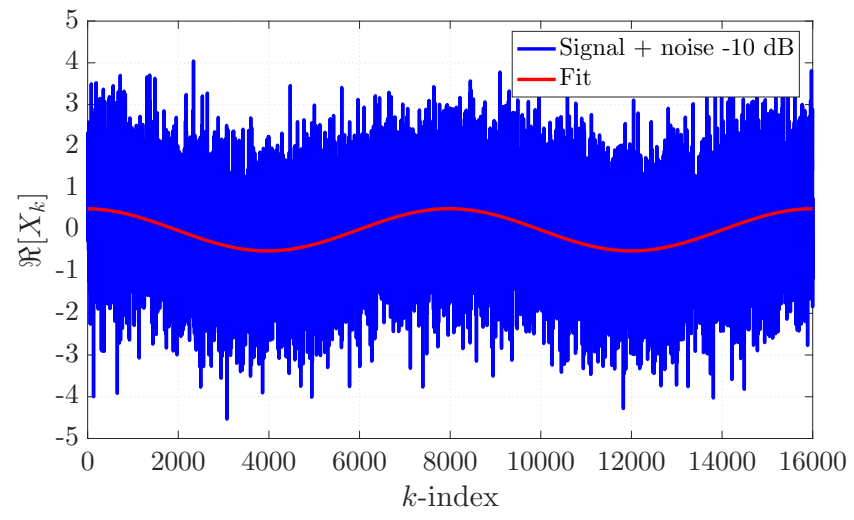

Fig. 3. Real part of noisy generated signal over a frequency bandwidth $\Delta f=$ $2 \delta f$ and containing with $\mathrm{N}=16000$ uncorrelated samples for a $-10 \mathrm{~dB}$ SNR level.

The sine wave amplitude $A\left(f_{0}, \theta_{\mathrm{T}}\right)$ is estimated from the difference, over the previously mentioned frequency range, of the complex scattering parameters $S_{\mathrm{T}}\left(f, \alpha_{\mathrm{st}}, \theta_{\mathrm{T}}\right)$ and $S\left(f, \alpha_{\text {st }}\right)$, such as :

$$
\begin{gathered}
\operatorname{argmin}_{A\left(f_{0}, \theta_{\mathrm{T}}\right), \phi_{0}} \mid A\left(f_{0}, \theta_{\mathrm{T}}\right) \exp \left[-\mathrm{j}\left(2 \pi \frac{f_{0}+f_{k}}{\delta f}-\phi_{0}\right)\right] \\
-\left(S_{\mathrm{T}}\left(f_{0}+f_{k}, \alpha_{\mathrm{st}}, \theta_{\mathrm{T}}\right)-S\left(f_{0}+f_{k}, \alpha_{\mathrm{st}}\right)\right) \mid \\
\text { with } f_{k}=k \delta f_{s}, k=-(N-1) / 2, \ldots, 0, \ldots,(N-1) / 2
\end{gathered}
$$

The antenna gain $G_{\text {ant }}$ and impedance mismatch $S_{\mathrm{FS}}$ may significantly vary over the considered bandwidth of analysis $\Delta f$. In this case, the estimation of the sine wave envelope may be carried out, once the difference of $S$ parameters is compensated for these factors.

\section{NUMERICAL ANALYSIS}

The purpose of this section is to numerically analyze how the frequency range $\Delta f$ and the frequency step $\delta f_{s}$ impact the accuracy of the extracted amplitude $A$, and thus the retrieved RCS.

For this parametric study a noiseless sine wave of period $\delta f$ is constructed over a variable frequency band $\Delta f$ and considering several numbers of frequency steps $N . \delta f$ is chosen to be coherent with actual measurement distances within the RC. Then, a white Gaussian noise (WGN) $n(f)$ is added to this sine function in order to emulate the backscattered response of the RC $x(f)$ (Fig. 3) such as :

$$
x_{k}=A \exp \left(\mathrm{j} 2 \pi \frac{k \delta f_{s}}{\delta f}\right)+n_{k}
$$

where $k=0, \ldots,(N-1)$. The amplitude of $n$ is set according to a desired Signal-to-Noise Ratio (SNR) defined as

$$
\mathrm{SNR}=\frac{\sum_{k=0}^{N-1}\left|A \exp \left(\mathrm{j} 2 \pi \frac{k \delta f_{s}}{\delta f}\right)\right|^{2}}{\sum_{k=0}^{N-1}\left|n_{k}\right|^{2}}
$$

The accuracy of the sine wave amplitude extraction from the noisy signal $x(f)$ is then estimated when varying several

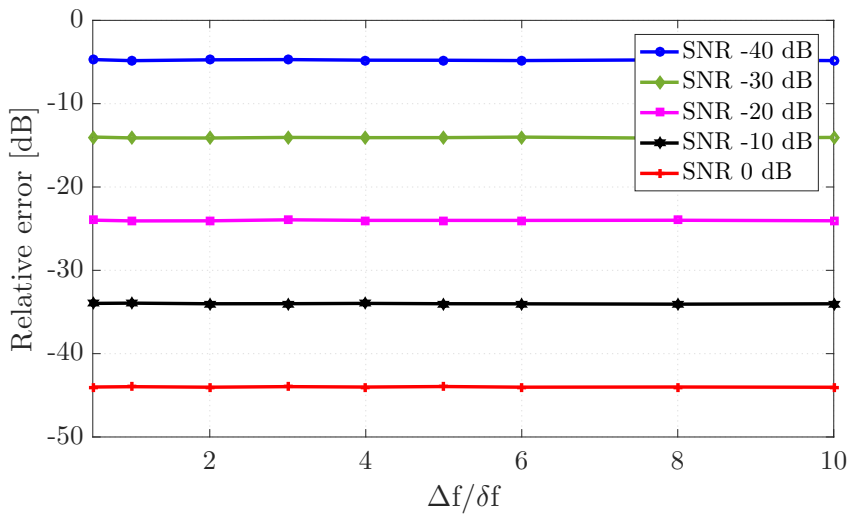

Fig. 4. Relative error on the estimated sine wave amplitude $A$ as a function of the normalized frequency bandwidth $\Delta f / \delta f$, averaged over 10000 noise random draws, for $N=16000$ uncorrelated frequency samples and various SNR values.

characteristic parameters. In a first part, all noise samples are considered uncorrelated, meaning that the RC coherence bandwidth is artificially set to zero, whereas correlated samples are considered in a second part to emulate the actual coherence bandwidth of the RC.

\section{A. Vanishing coherence bandwidth (uncorrelated samples)}

Firstly, several frequency ranges $\Delta f$ are considered. As the sine wave period $\delta f$ is the representative quantity of the signal variation, the considered frequency ranges are expressed versus $\delta f(\Delta f=$ $\{0.5 \delta f, 1 \delta f, 2 \delta f, 3 \delta f, 4 \delta f, 5 \delta f, 6 \delta f, 8 \delta f, 10 \delta f\})$. For all cases, the number of frequency steps (equally spaced within $\Delta f$ ) is constant and set to 16000 . The relative error on the evaluated amplitude of the sine wave, averaged over 10000 random draws of $x(f)$, is presented in Fig. 4 for an SNR ranging from $-40 \mathrm{~dB}$ to $0 \mathrm{~dB}$. We show that the number of sine periods $(\delta f)$ contained in the $\Delta f$ does not affect the accuracy, for a constant $N=16000$. Also and as expected, the error decreases for higher SNR values; indeed, the relative error decreases in the same proportion as the SNR.

Then, the frequency bandwidth is kept constant so that $\Delta f=2 \delta f$ and the number of frequency steps $N$ is modified from 125 to 16000 . The relative error on the evaluated amplitude averaged over 10000 random draws is presented in Fig. 5 as a function of the SNR. We show here that the more the number of points, the lower the error; more precisely, the relative error varies versus $N$ as $-20 \times \log (\sqrt{N})$ as expected with uncorrelated samples. This result suggests that one should choose the largest possible number of frequency points in order to enhance the accuracy. However, a vanished coherence bandwidth is considered here, which corresponds to an infinite quality factor $Q$ of the cavity, neglecting the losses induced by the RC and the objects loading it (including the antenna).

\section{B. Realistic coherence bandwidth (correlated samples)}

In practice, the finite $Q$ of the RC implies that the frequency samples are correlated if the frequency step is lower than the 


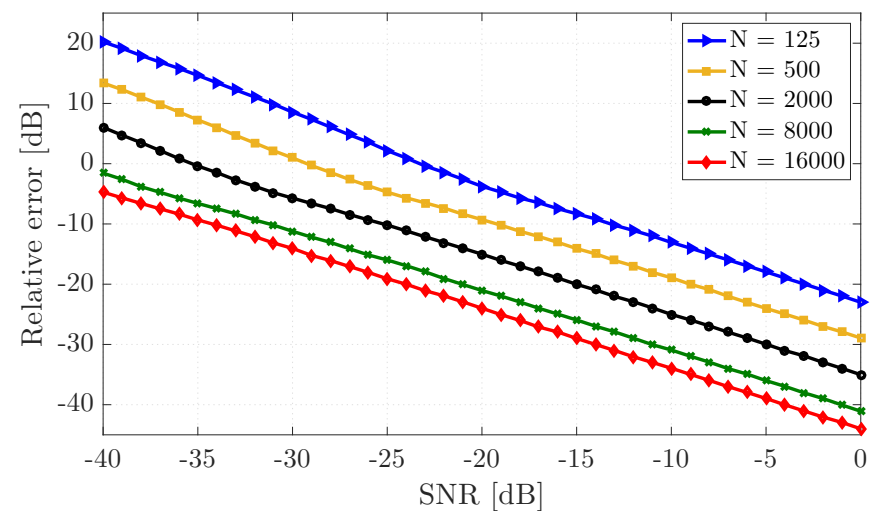

Fig. 5. Relative error on the estimated sine wave amplitude as a function of the SNR, for $\Delta f=2 \delta f$, averaged over 10000 noise random draws, for various numbers of frequency steps $N$.

coherence bandwidth. The effect of correlation is investigated in this part. We assume a constant bandwidth $\Delta f=2 \delta f$, and consider a constant sample size equal to 16000 . The correlated noise samples are generated using a first order Auto Regressive Moving Average (ARMA) process meaning that each sample $n_{k}$ is defined as

$$
n_{k}=c+\rho n_{k-1}+\epsilon_{k}
$$

where $\rho$ is the ARMA coefficient ranging from 0 (no correlation) to 1 (full correlation), the constant $c$ is fixed to 0 as the noise is centered, and $\epsilon_{k}$ corresponds to a WGN whose variance $\sigma^{2}$ is chosen in regard to the one of $n_{k}$ namely $\sigma_{n}^{2}$ as $\sigma^{2}=\left(1-\rho^{2}\right) \sigma_{n}^{2}$. The effective sample size $N_{\text {eff }}$ is then a fraction of the actual sample size $N$ depending on $\rho$ according to

$$
N_{\text {eff }}=\frac{1-\rho}{1+\rho} N .
$$

Though it has been shown [17] that the first order ARMA model is only valid to describe correlation between samples in $\mathrm{RC}$ for $\rho<0.55$ (or approximately), we highlight here the effect of the first order correlation up to $\rho=0.9$, neglecting the second order correlation, that would even increase the correlation effect. This choice permits to highlight the correlation effect on the extraction precision using a single parameter that is the correlation coefficient $\rho$.

The relative error on the estimated sine wave amplitude is presented in Fig. 6 as a function of the number of equally spaced samples actually taken within the 16000 frequency samples for different levels of correlation expressed in terms of $N_{\text {eff }}$. A few conclusions can be drawn from Fig. 6. First, for a fixed number of samples, e.g., 16000, the error is lower for larger $N_{\text {eff }}$ values. Second, for a given $N_{\text {eff }}$, the error does almost not decrease anymore when the number of samples becomes larger than $N_{\text {eff. }}$. However, although it seems useless to use more samples than $N_{\text {eff }}$, it does not alter the results neither.

From this section, we can conclude that the fundamental parameter to enhance the accuracy is the effective sample size $N_{\text {eff. }}$. For practical measurement, $\Delta f$ needs to be chosen as

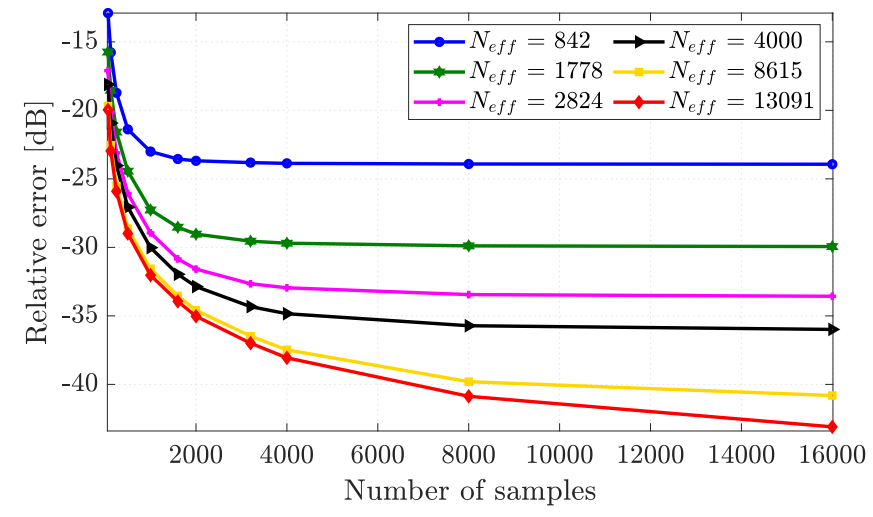

Fig. 6. Relative error on the estimated sine wave amplitude, averaged over 10000 random draws, for various effective sample sizes.

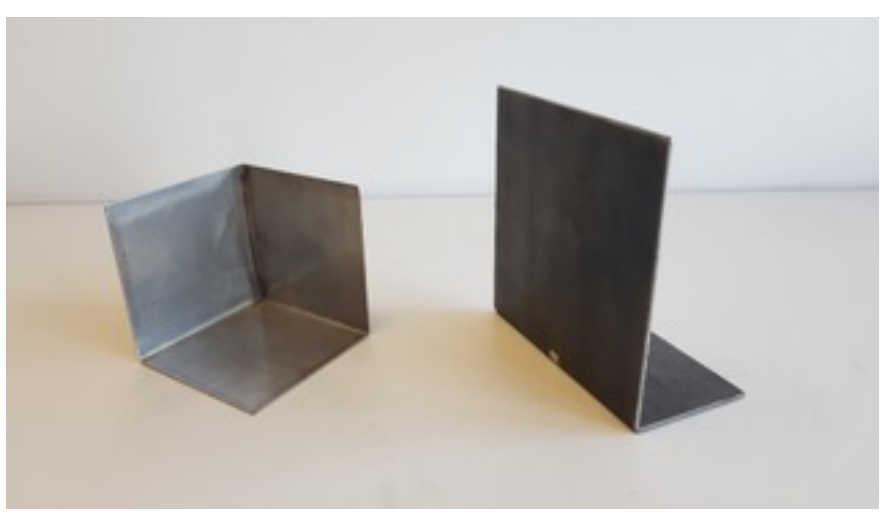

Fig. 7. Picture of the metallic $100 \mathrm{~mm} \times 100 \mathrm{~mm} \times 100 \mathrm{~mm}$ dihedral (left) and the $148 \mathrm{~mm} \times 151 \mathrm{~mm}$ metallic plate (right).

large as possible to increase $N_{\text {eff }}$ in relation with the coherence bandwidth. However, as this also implies a "frequency averaging" of the retrieved RCS according to the RCS variation versus frequency, a trade-off has to be made.

\section{RCS Measurement SETUPS}

\section{A. Presentation of the targets}

Two targets are considered in order to validate the RCS measurement method within RC. The first one is a metallic plate that has been bended at 90 degrees, forming an horizontal foot of $5 \mathrm{~cm}$ width (Fig. 7) so that it can stand in a vertical position. The dimensions of the vertical part are $148 \mathrm{~mm}$ (horizontally) $\times 151 \mathrm{~mm}$ (vertically). The second target is a trihedral of dimensions $100 \mathrm{~mm} \times 100 \mathrm{~mm} \times 100 \mathrm{~mm}$; placed on one face during measurement, it behaves as a dihedral. The RCS patterns of both targets are first measured in an anechoic chamber $(\mathrm{AC})$ in order to serve as a reference for further comparisons with the introduced RC technique, even if such measurements are never free from measurement errors (in particular due to positioning inaccuracy).

\section{B. Anechoic Chamber}

The RCS measurements are performed in the AC of IETR using a rotationally driven positioning mast for the target and a mono-static measuring system composed of two X-band horn 


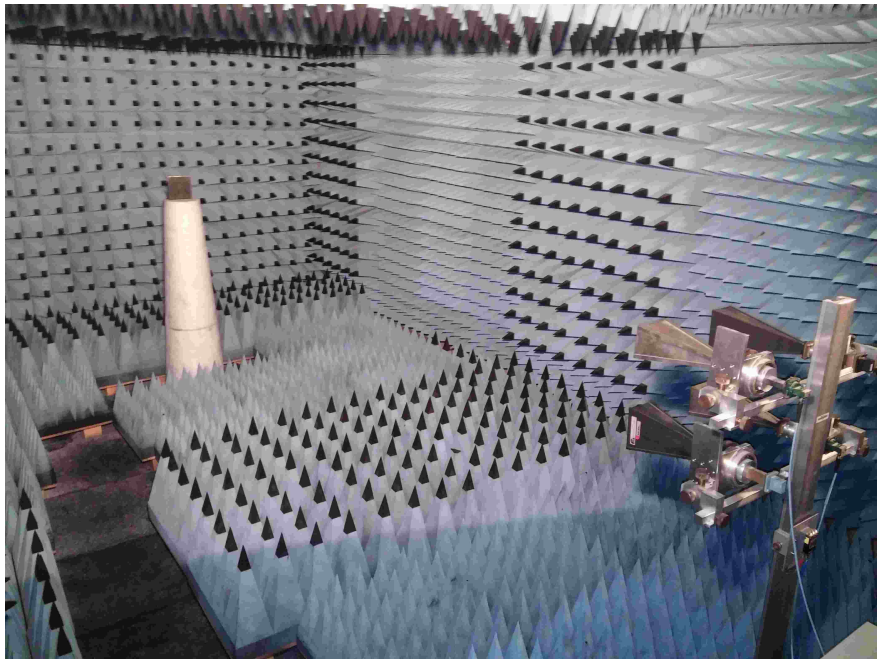

Fig. 8. Test set-up for RCS measurement in IETR anechoic chamber.

antennas, one transmitting and the other receiving, connected to a VNA to measure the S-parameters between both antennas (see Fig. 8). Beforehand, it is advisable to take a measurement in the empty chamber to subtract this response from the measurements with the target. Then, a first step consists of calibrating the measurement via a reference target placed on the mast at 4 meters from the antennas and at the same height as them, and by rotating the mast to search the maximal RCS of the reference target. In a second step, the target under test is inserted and, at each target position, a measurement over a wide frequency band ( 8 to $12 \mathrm{GHz}$ ) is carried out in order to be able to precisely spot the target signature on the temporal response obtained by inverse Fourier transform applied to the frequency measurement. It allows to perform a time gating operation to only isolate the response of the target and improve the SNR.

\section{Reverberation Chamber}

Two RCs are used to perform RCS measurements: the one at IETR and the one at ESYCOM. They are both oversized metallic cavities made in aluminium but differ in size and mechanical stirrer shape. The IETR one is indeed larger than the ESYCOM one and theoretically better stirs the field. The same measurement setup is used (Fig. 1) in both RCs including a transmitting/receiving horn antenna whose reflection coefficient is measured using a VNA. The horn antenna, the target and the stirrer are aligned in both cases. The far-field condition at $10 \mathrm{GHz}$ would require $R=3 \mathrm{~m}$ for the metallic plate. This condition is verified at IETR whereas $R$ is reduced to $2.35 \mathrm{~m}$ at ESYCOM due to limited space. This could lead to measurement inaccuracy, especially regarding the level of the lowest extreme values. The main properties of the two RCs as well as the measurement details are presented in Tab. I.

\section{Radar Cross Section Pattern of a metallic PLATE}

In this section, the RCS of the metallic plate measured within the two RCs are presented and compared to the one
TABLE I

MAIN PROPERTIES OF THE TWO RCS AND MEASUREMENT SETUP.

\begin{tabular}{|l|l|l|}
\hline Properties & IETR & ESYCOM \\
\hline Size $(l * w * h)$ & $8.70 \times 3.70 \times$ & $2.95 \times 2.75 \times$ \\
& $2.90 \mathrm{~m}^{3}$ & $2.35 \mathrm{~m}^{3}$ \\
\hline Total volume & $93.3 \mathrm{~m}^{3}$ & $19.1 \mathrm{~m}^{3}$ \\
\hline Estimated LUF & $200 \mathrm{MHz}$ & $400 \mathrm{MHz}$ \\
\hline Measured frequency & $9.75 \mathrm{GHz}$ & $9.75 \mathrm{GHz}$ \\
band & $10.25 \mathrm{GHz}$ & $10.25 \mathrm{GHz}$ \\
\hline Q factor at $10 \mathrm{GHz}$ & 10000 & 25000 \\
\hline Coherence bandwidth & $100 \mathrm{kHz}$ & $400 \mathrm{kHz}$ \\
$(10 \mathrm{GHz})$ & $\left(N_{\mathrm{eff}}=5000\right)$ & $\left(N_{\text {eff }}=1250\right)$ \\
\hline Uncorrelated stirrer po- & 51 & 7 \\
sitions $N_{\alpha_{\text {st }}^{u}}(10 \mathrm{GHz})$ & & \\
\hline Distance $R$ & $2.95 \mathrm{~m}$ & $2.46 \mathrm{~m}$ \\
\hline
\end{tabular}

obtained through measurement in AC. As no calibration measurement has been performed (using a standard target), the obtained results have been normalized so that the maximal RCS value (corresponding to the target position $\theta_{\mathrm{T}}=0^{\circ}$ ) is equal to the theoretical one $\sigma=\frac{4 \pi S_{p}^{2} f^{2}}{c^{2}}$ (with $S_{p}$ the target section and $c$ the light velocity) for the metallic plate [18]. The choice of this target shape is of particular interest to test our measurement technique accuracy due to the large variation of the RCS amplitude between the main lobe and the side ones.

\section{A. RCS for a fixed stirrer position}

The RCS of the metallic plate has firstly been extracted for fixed stirrer positions. The RCS patterns obtained with three arbitrary chosen stirrer positions $\left(\alpha_{\mathrm{st}}=36^{\circ}, 90^{\circ}, 180^{\circ}\right)$ at IETR (Fig. 9) and at ESYCOM (Fig. 10) are compared to the one measured in AC. A few comments can be drawn from these results. First we can see that the overall RCS pattern is well retrieved in both RCs for all considered stirrer positions. Second, the agreement between RC and AC measurements is particularly good for the main RCS lobe, that corresponds to the highest SNR levels. The accuracy on the level of the secondary lobes is however better in the IETR RC; this more accurate sine wave amplitude extraction can be explained by the higher number of uncorrelated frequency samples $N_{\text {eff }}$ in IETR RC due to the smaller coherence bandwidth (Tab. I). Finally, the three RCS patterns obtained for various stirrer positions are not identical, implying that the stirrer has an impact on the retrieved RCS.

\section{B. Impact of mechanical stirring}

In this part, we evaluate the impact of the mechanical stirring on the RCS pattern measurement. As introduced in section II, the evaluation of the RCS relies on the extraction of the sine signal amplitude from the difference of the S-parameters with and without the target, i.e., $S_{\mathrm{T}}\left(f_{0}, \alpha_{\mathrm{st}}, \theta_{\mathrm{T}}\right)-S\left(f_{0}, \alpha_{\mathrm{st}}\right)$, once it is compensated for antenna gain and mismatches. Fig. 11 shows the waveform when the incident wave vector is normal to the plate surface $\left(\theta_{\mathrm{T}}=0^{\circ}\right)$, and the stirrer position is at its initial position (labelled as $\alpha_{\mathrm{st}}=0^{\circ}$ ). This signal shows indeed an oscillatory pattern expressed by the first term of (6), partly hidden by a pseudo-noise interfering signal related to the second term of (6). To highlight the impact 


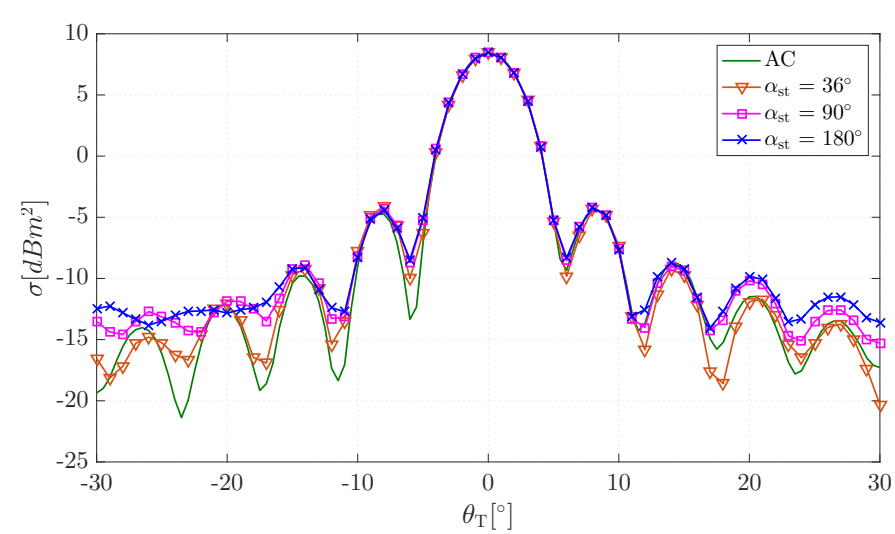

Fig. 9. RCS pattern at $10 \mathrm{GHz}$ of the metallic plate measured in IETR RC for three different stirrer positions compared to the one measured in $\mathrm{AC}$.

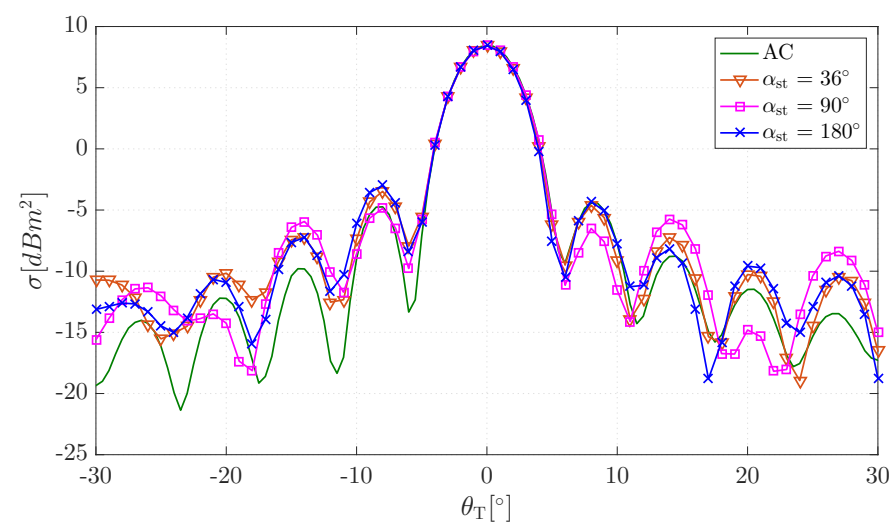

Fig. 10. RCS pattern at $10 \mathrm{GHz}$ of the metallic plate measured in ESYCOM $\mathrm{RC}$ for three different stirrer positions compared to the one measured in $\mathrm{AC}$.

of mechanical stirring, we show the average over a stirrer rotation $\left\langle S_{\mathrm{T}}\left(f_{0}, \alpha_{\mathrm{st}}, \theta_{\mathrm{T}}\right)-S\left(f_{0}, \alpha_{\mathrm{st}}\right)\right\rangle_{\alpha_{\mathrm{st}}}$ (green curve) of the difference between both measured reflection coefficients, with and without the target. We can see that it permits to reduce the noise $n\left(f_{0}, \alpha_{\mathrm{st}}, \theta_{\mathrm{T}}\right)$ in (6) so that the backscattered ballistic wave (red curve) may be extracted with higher accuracy from that signal. It has to be noted that the sine wave amplitude $A\left(f_{0}, \theta_{\mathrm{T}}\right)$ appears to be nearly constant over the considered $\Delta f(500 \mathrm{MHz})$. Besides, it has been verified that the noise signal, calculated by subtracting the retrieve sine wave signal to the difference between the S-parameters with and without the target, follows a normal distribution as supposed theoretically and in the numerical analysis.

To highlight the impact of such averaging on the retrieved SNR, as defined in (10), Fig. 12 and Fig. 13 show the SNR calculated from the RCS measurement of the metallic plate, in IETR RC and ESYCOM RC, respectively. It is presented as a function of the target position $\theta_{\mathrm{T}}$. The SNR for fixed $\alpha_{\mathrm{st}}$ are represented by the grey curves. As expected, it is maximum at $\theta_{\mathrm{T}}=0^{\circ}$ and it decreases following the RCS pattern. The average of these SNR patterns is represented by the solid black curve and varies between $-35 \mathrm{~dB}$ and $-9 \mathrm{~dB}$ at IETR RC and between $-30 \mathrm{~dB}$ and $-6 \mathrm{~dB}$ at ESYCOM RC. The SNR for $\theta_{\mathrm{T}}=0^{\circ}$ is higher in ESYCOM RC as the distance $R$ between the antenna and the target is smaller in this cavity. Finally,

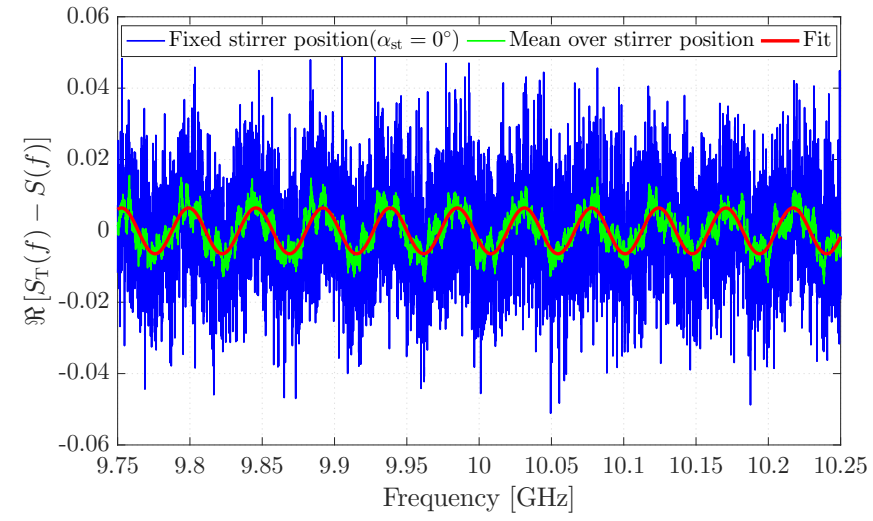

Fig. 11. Waveform versus frequency of the real part of the difference between the measured reflection coefficients without the target, and with the target (metallic plate) at $\theta_{\mathrm{T}}=0^{\circ}$ in IETR RC. The blue curve corresponds to $\alpha_{\mathrm{st}}=0^{\circ}$, the green curve to the average over a stirrer rotation $\langle\cdot\rangle_{\alpha_{\mathrm{st}}}$, and the red curve to the estimated sinusoidal component.

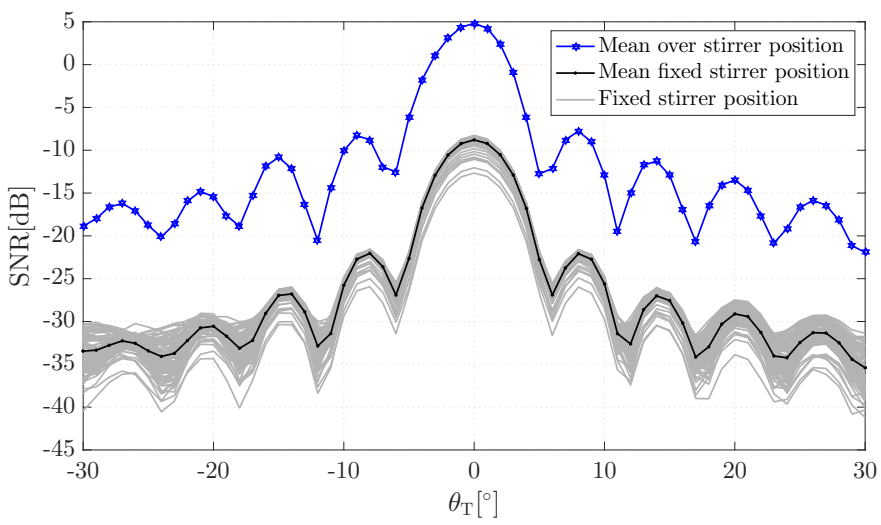

Fig. 12. SNR obtained from the RCS measurement of a metallic plate in IETR RC.

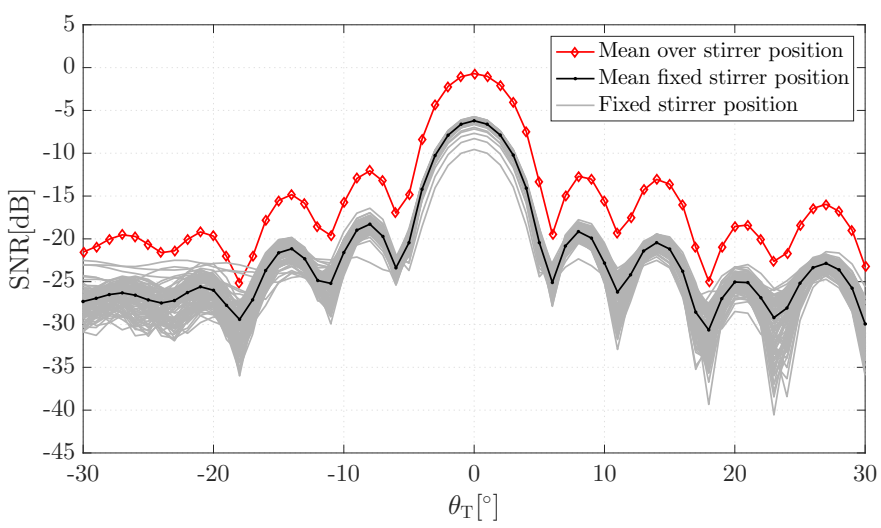

Fig. 13. SNR obtained from the RCS measurement of a metallic plate in ESYCOM RC.

the SNR associated to the RCS obtained after performing an average over the stirrer positions of the reflection coefficient difference is represented by the blue curve with hexagram markers and red curve with diamond markers, for IETR RC and ESYCOM RC, respectively. We observe that the SNR is strongly increased thanks to the stirring process in both RCs. The SNR enhancement varies between $13.1 \mathrm{~dB}$ and $17.5 \mathrm{~dB}$ 


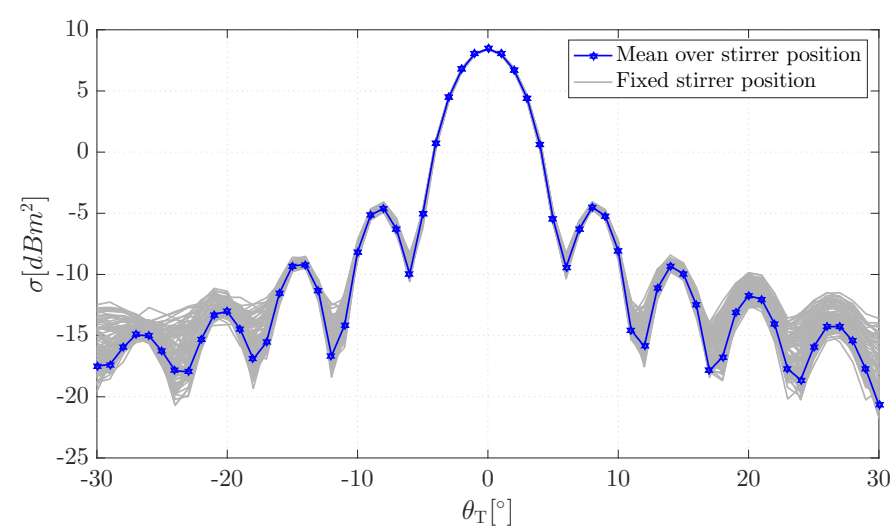

Fig. 14. RCS pattern at $10 \mathrm{GHz}$ of the metallic plate measured in IETR RC obtained with and without applying the stirring process.

in IETR RC, with a mean value of $13.6 \mathrm{~dB}$ whereas the mean SNR enhancement only reaches $5.5 \mathrm{~dB}$ in ESYCOM RC with a variation range between $5.1 \mathrm{~dB}$ and $8.9 \mathrm{~dB}$. This highlights the difference in terms of stirring quality between both RCs. Indeed, the SNR enhancement $\Delta_{\text {SNR }}$ is linked to the total number of uncorrelated stirrer positions $N_{\alpha_{\mathrm{st}}}^{u}$ as

$$
\Delta_{\mathrm{SNR}}=10 \times \log \left(N_{\alpha_{\mathrm{st}}}^{u}\right) .
$$

Although the measurements have been performed for 100 stirrer positions in both cavities, they are not likely to be all uncorrelated. The numbers of uncorrelated stirrer positions at $10 \mathrm{GHz} N_{\alpha_{\text {st }}}^{u}$, evaluated in both RCs using the correlation technique and (12) applied on the measured reflection coefficients in the empty RCs, are indicated in Tab. I. It has to be noticed that the estimation of a very low number of uncorrelated stirrer positions as in ESYCOM RC is associated with a large uncertainty due to the small size of the effective sample.

According to the estimated number of uncorrelated stirrer positions in both RCs, (13) leads to an expected SNR improvement of $17.1 \mathrm{~dB}$ in IETR RC and $8.5 \mathrm{~dB}$ in ESYCOM RC. These theoretical values, obtained from a formula valid for large independent sample numbers, are of the order of magnitude of the best obtained SNR enhancements in both cavities, and confirm the advantage of the stirring process to improve the RCS extraction accuracy.

The RCS patterns of the metallic plate obtained for each $\alpha_{\text {st }}$ taken independently are compared to the one obtained after averaging the reflection coefficient difference over a stirrer rotation in Fig. 14 and Fig. 15 at IETR and ESYCOM, respectively. It is observed that the discrepancy between the RCS retrieved for single $\alpha_{\text {st }}$ can reach up to $10 \mathrm{~dB}$ for low RCS levels $\left(\left|\theta_{\mathrm{T}}\right|>15^{\circ}\right)$ due to the difficulty to extract a low amplitude sine wave signal among a noisy one.

\section{Comparison with AC measurement}

The RCS pattern of the metallic plate retrieved from RC measurements (including the stirring process) is now compared to the one measured in $\mathrm{AC}$, considered as a reference measurement. Results are presented in Fig. 16. It shows a

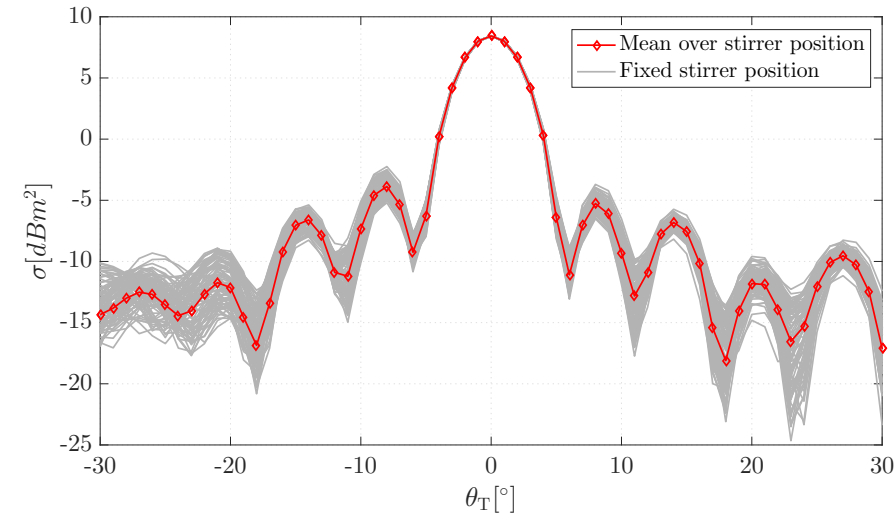

Fig. 15. RCS pattern at $10 \mathrm{GHz}$ of the metallic plate measured in ESYCOM $\mathrm{RC}$ obtained with and without applying the stirring process.

good overall agreement between both measurement methods. In order to quantify the difference between the RCS pattern evaluated in the $\mathrm{AC}$, considered as the reference, and the ones extracted from measurements in both RCs, the relative error is calculated as follows:

$$
E r r_{\text {rel }}=\left\langle\left|\sigma_{\mathrm{T}}^{A C}\left(f_{0}, \theta_{\mathrm{T}}\right)-\sigma_{\mathrm{T}}^{R C}\left(f_{0}, \theta_{\mathrm{T}}\right)\right| / \sigma_{\mathrm{T}}^{A C}\left(f_{0}, \theta_{\mathrm{T}}\right)\right\rangle_{\theta_{\mathrm{T}}} .
$$

where $\langle.\rangle_{\theta_{\mathrm{T}}}$ indicates a mean over the target angles. The obtained values in $\mathrm{dB}$, using the conversion formula $E r r_{\mathrm{dB}}=$ $10 \times \log \left(E r r_{\text {rel }}+1\right)$, are of $2.4 \mathrm{~dB}$ with ESYCOM RC and $0.9 \mathrm{~dB}$ with IETR RC. Despite this overall good agreement, the measure in IETR RC is closer to the $\mathrm{AC}$ measurement than the measure in ESYCOM RC. In particular, the side lobes level is better evaluated as well as the low RCS values. Three main reasons can explain this accuracy difference. First of all, due to the limited available space, the distance between the antenna and the target is too short in ESYCOM RC according to farfield condition. Second, the coherence bandwidth, linked to the quality factor, is larger in this cavity, leading to a smaller number independent frequency realizations over the measured frequency band and more difficulties to get rid of the noise to extract the signal of interest. Finally, the stirring process is more effective in IETR RC with a higher number of stirrer independent positions. Lowering the Q-factor of the RC could be seen as an alternative solution to increase the SNR level. Nevertheless, according to a fixed stirrer position, the ratio of the Q-factor over $N_{\text {eff }}$ (12) would remain constant. However it would yield a negative impact on the mechanical stirrer efficiency. It has to be noticed that the patterns are not strictly symmetric for all measurements implying a potential slight misalignment of the target. Indeed, in both RCs, the excitation antenna and the rotating mast have been set up manually without dedicated device to ensure a good alignment: a more sophisticated measurement setup, as classically used in ACs for RCS measurements, would help to increase the measures accuracy.

\section{Method validation with metallic dihedral}

This part is dedicated to the validation of the introduced technique on a second target of more complex shape and 


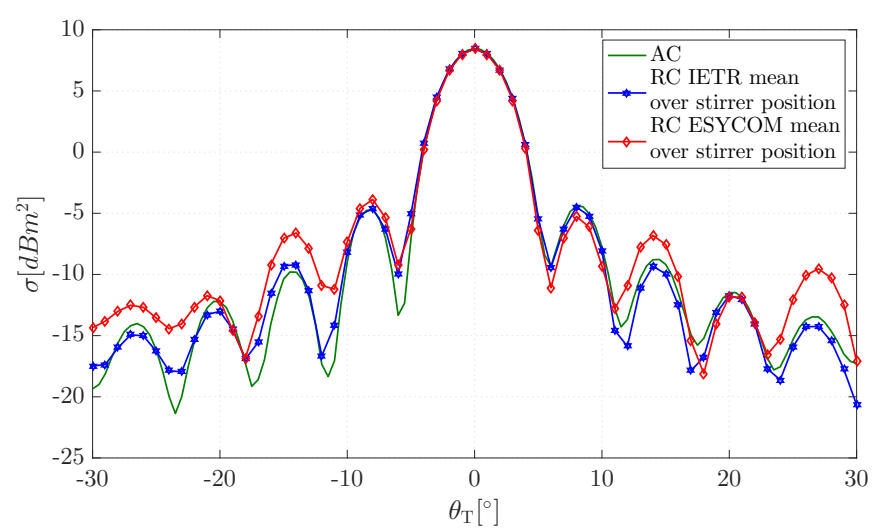

Fig. 16. RCS pattern at $10 \mathrm{GHz}$ for a metallic plate obtained in IETR and ESYCOM RCs using the stirring process, compared with RCS pattern measured in AC.

generating multiple reflection phenomena: the dihedral presented in section IV-A. The same measurement setup is used with identical frequency range and frequency steps. Some results are skipped for brevity but the interest of the stirring process is still highlighted. The RCS patterns of the dihedral target measured in IETR and ESYCOM RCs are presented in Fig. 17 and Fig. 18, respectively. The obtained RCS have been normalized so that their maximal value (obtained at the target position $\theta_{\mathrm{T}}=0$ ) is equal to the theoretical one that is $\sigma=\frac{8 \pi S_{\mathrm{d}}^{2} f^{2}}{c^{2}}$ (with $S_{\mathrm{d}}$ the surface of one side of the dihedral and $c$ the light velocity). The RCS estimated from single stirrer position measurements (grey curves) is compared to the one obtained after applying the stirring process. First, we notice that the discrepancies between all $\alpha_{\mathrm{st}}$ is much lower than for the plate due to the higher RCS levels in this angular range. Indeed, it reaches a maximum of $1.5 \mathrm{~dB}$ for the lowest RCS in ESYCOM RC whereas it is kept under $1 \mathrm{~dB}$ in IETR RC. Fig. 19 shows the RCS pattern of the metallic dihedral, obtained through an average over stirrer positions in both RCs, at IETR (blue curve) and ESYCOM (red curve) and compared to the one obtained through measurement performed in the $\mathrm{AC}$ (green curve), considered as a reference measurement for the metallic dihedral. For this target, we also see a good agreement between the three measurements, with a maximum difference equal to $1.5 \mathrm{~dB}$ and similar overall $\operatorname{Err}_{\mathrm{dB}}$ values of $0.37 \mathrm{~dB}$ in ESYCOM RC against $0.43 \mathrm{~dB}$ in IETR RC. The accuracy of target alignment (performed by hand) may also partly explain the residual discrepancy as well as the neglected potential specular reflections between the target, the wall and the measurement antenna.

\section{CONCLUSION}

The method of RCS measurement in RC presented in this paper exploits the diffuse field properties within well-operating RCs to extract the target signature from the difference between measured scattering parameters with and without the target. This paper is based on a preliminary demonstration of the ability of such approach to retrieve the RCS from RC measurement, but it generalizes the initially proposed theory by taking the stirrer position into account and taking advantage of

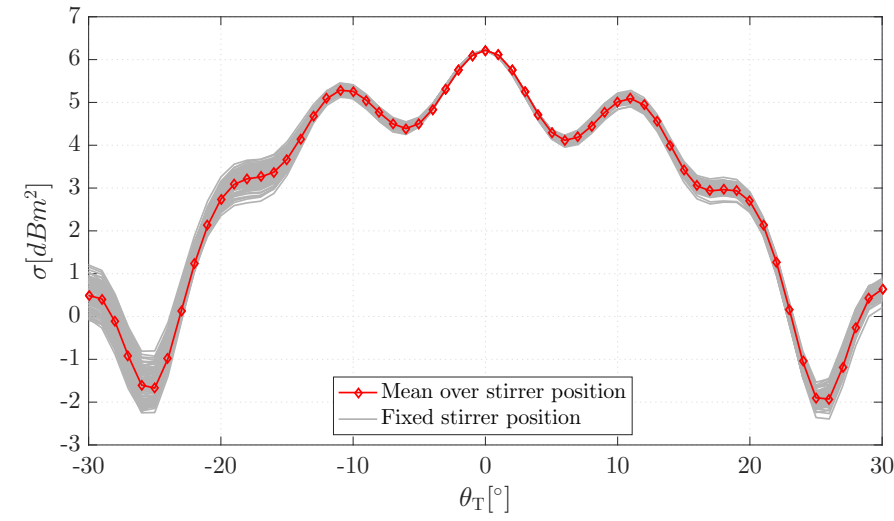

Fig. 17. RCS pattern at $10 \mathrm{GHz}$ for a metallic dihedral obtained in IETR RC with and without applying the stirring process.

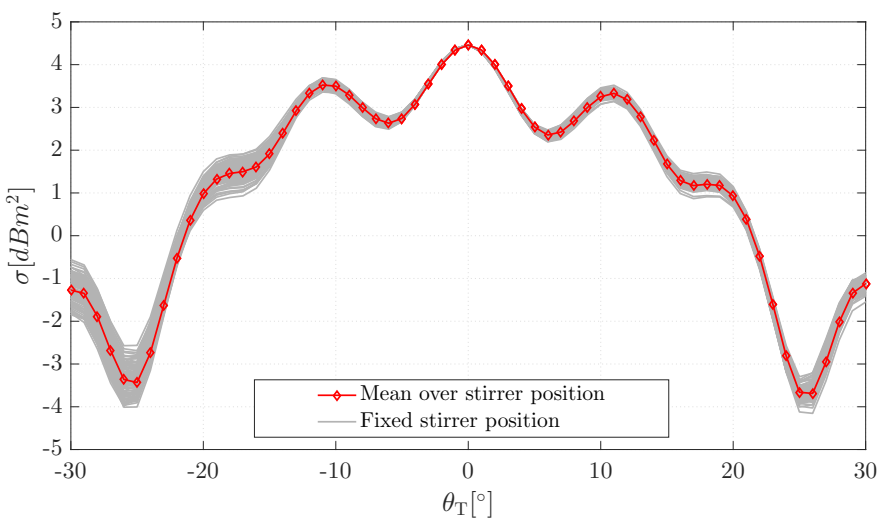

Fig. 18. RCS pattern at $10 \mathrm{GHz}$ for a metallic dihedral obtained in ESYCOM $\mathrm{RC}$ with and without applying the stirring process.

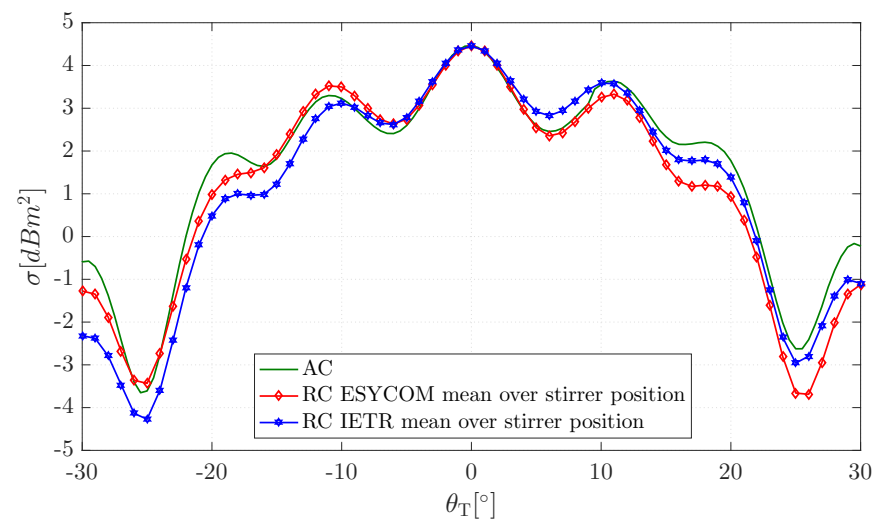

Fig. 19. RCS pattern at $10 \mathrm{GHz}$ for a metallic dihedral obtained in IETR and ESYCOM RCs applying the stirring process, compared with RCS pattern measured in AC.

the stirring process to enhance the extraction accuracy. Indeed, the interest of operating a stirring process (mechanical here) in order to increase the SNR in regard to the signal of interest has been demonstrated.

For a deeper view of the RCS extraction method accuracy, a numerical analysis has been performed in order to study the impact of several parameters including the frequency range, the frequency step, and SNR. It has been pointed out that the main limitations are due to the properties of the RC itself, 
namely its coherence bandwidth and number of uncorrelated stirrer positions.

The introduced method has been validated by measuring the RCS pattern of two different metallic targets, namely a rectangular plate and a dihedral target. These measurements have been performed in two RCs of different characteristics (different size and stirring efficiency). Both results are very close and in good agreement with the reference measurement performed within an AC.

\section{ACKNOWLEDGMENT}

The authors would like to thank the Direction Générale de l'Armement (DGA) of the French Ministry of the Armed Forces to financially support this work. This work was also supported in part by the European Union through the European Regional Development Fund, in part by the Ministry of Higher Education and Research, in part by the Région Bretagne, and in part by the Département d'Ille et Vilaine and Rennes Métropole, through the CPER Project SOPHIE/STIC \& Ondes.

\section{REFERENCES}

[1] P. Corona, G. Latmiral, E. Paolini, L. Piccioli, Use of a reverberating enclosure for measurements of radiated power in the microwave ranges, IEEE Trans. Electromagn. Compat. vol. 18, no. 2, pp. 54-59, May 1976.

[2] K. Rosengren, P-S. Kildal, Study of distributions of modes and plane waves in reverberation chamber for characterization of antennas in multipath environment, MOTL vol. 30, no. 6, pp. 386-391, Sep. 2001.

[3] G. Lerosey, J. de Rosny, Scattering cross section measurement in reverberation chamber, IEEE Trans. Electromagn. Compat. vol. 49, no. 2, pp. 280-284, May 2007.

[4] A. Cozza, Emulating an anechoic environment in a wave-diffusive medium through an extended time-reversal approach, IEEE Trans. Antennas Propagat. vol. 60, no.8, pp 3838-3852, Aug. 2012.

[5] C. Lemoine, E. Amador, P. Besnier, J.M. Floc'h, Antenna directivity measurement in reverberation chamber from Rician K-factor estimation, IEEE Trans. Antennas Propagat. vol. 61, no. 10, pp. 5307-5310, Oct. 2013.

[6] M. Garcia-Fernandez, D. Carsenat, C. Decroze, Antenna gain and radiation pattern measurements in reverberation chamber using Doppler effect, IEEE Trans. Antennas Propagat. vol. 62, no.10, pp 5389-5394, Oct. 2014.

[7] $\mathrm{Q}$. Xu et al., 3-D antenna radiation pattern reconstruction in a reverberation chamber using spherical wave decomposition, IEEE Trans. Antennas Propagat. vol. 65, no.4, pp 1728-1739, Apr. 2017.

[8] A. Soltane, G. Andrieu, E. Perrin, C. Decroze, A. Reineix, Antenna Radiation Pattern Measurement in a Reverberating Enclosure Using the Time-Gating Technique, IEEE Antennas and Wireless Propagation Letters, vol. 19, no. 1, pp 183-187, 2020.

[9] A. Soltane, G. Andrieu, A. Reineix, Monostatic Radar Cross-Section Estimation of Canonical Targets in Reverberating Room Using TimeGating Technique, 2018 International Symposium on Electromagnetic Compatibility (EMC Europe), pp. 355-359, Aug. 2018.

[10] P. Besnier, J. Sol, S. Méric, Estimating radar cross-section of canonical targets in reverberation chamber, 2017 International Symposium on Electromagnetic Compatibility (EMC EUROPE), pp 1-5, Sep. 2017.

[11] A. Reis, F. Sarrazin, E. Richalot and P. Pouliguen, Mode-Stirring Impact in Radar Cross Section Evaluation in Reverberation Chamber, 2018 International Symposium on Electromagnetic Compatibility (EMC EUROPE), pp 875-878, Aug. 2018.

[12] P. Besnier, B. Demoulin, Electromagnetic reverberation chambers, ISTE Wiley\& Sons, 2011

[13] C. Lemoine, P. Besnier, M. Drissi, Investigation of Reverberation Chamber Measurements Through High-Power Goodness-of-Fit Tests, IEEE Trans. Electromagn. Compat. vol. 49, no. 4, pp 745-755, Nov. 2007.

[14] G. Andrieu, Electromagnetic Reverberation Chambers : Recent Advances and Innovative Applications, The Institute of Engineering and Technology (IET), 2020.
[15] A. Reis, F. Sarrazin, P. Pouliguen, J. Sol, P. Besnier and E. Richalot, Radar Cross Section Measurement within Reverberation Chamber: Stirrer Position Issues, 2020 European Conference on Antennas and Propagation (EuCAP), pp 1-4, Mar. 2020.

[16] M. Born, E. Wolf, Principles of optics: electromagnetic theory of propagation, interference and diffraction of light, Elsevier, 2013.

[17] C. Lemoine, P. Besnier, M. Drissi, Estimating the Effective Sample Size to Select Independent Measurements in a Reverberation Chamber, IEEE Trans. Electromagn. Compat. vol. 50, no. 2, pp. 227-236, May 2008.

[18] G.T. Ruck, D.E. Barrick, W.D. Stuart, C.K. Krichbaum, Radar Cross Section Handbook, Plenum Press, New York, 1970.

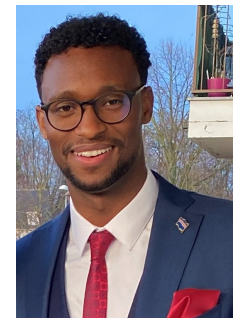

Ariston Reis was born in Praia, Cap-Verd, in 1991. $\mathrm{He}$ received the M.S. degree in biomedical engineering from University of Montpellier (Faculté des Sciences), Montpellier, France, in 2017. Since 2017, he has been working toward the Ph.D. degree at the Electronics, Communication Systems and Microsystems laboratory (ESYCOM), Université Gustave Eiffel, Champs-Sur-Marne, France. His current research activities are in the field of antenna characterization using the backscattering method, which consists in measuring the Radar Cross Section (RCS) in a mode-stirred reverberation chamber.

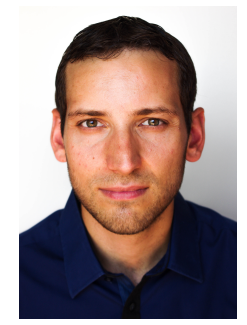

François Sarrazin received the M.S. degree in electronics and electrical engineering from Polytech'Nantes (Ecole polytechnique de l'université de Nantes), in 2010, and the Ph.D. degree from the Institute of Electronics and Telecommunications of Rennes (IETR), University of Rennes 1, in 2013. In 2014 , he worked as a post-doctorate fellow at the Royal Military College of Canada in Kingston, Ontario. From 2010 to 2014, his research was focused on antenna characterization using the Singularity Expansion Method (SEM) applied both in the time and the spatial domains. In 2015, he worked as a research engineer at the CEA-Léti in Grenoble. He did his research on electrically small frequencyagile antenna and radiation efficiency optimization. Since September 2016, he is an associate professor at the Université Gustave Eiffel where he joined the Electronics, Communication System and Microsystem laboratory (ESYCOM) to conduct his research. His research activities include reverberation chamber characterization, antenna efficiency measurement, contactless antenna characterization from RCS measurement and miniature antennas.

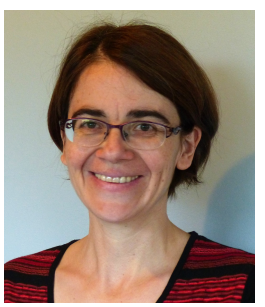

Elodie Richalot received the Diploma and $\mathrm{Ph} . \mathrm{D}$. degrees in electronics engineering from école nationale supérieure d'électrotechnique, d'électronique, d'informatique, d'hydraulique Toulouse, France, in 1995 and 1998, respectively. Since 1998, she has been with the Universite Gustave Eiffel, Champs-sur-Marne, France, where she became a Professor of electronics in 2010. Her current research activities in ESYCOM laboratory include modeling techniques, electromagnetic compatibility and reverberation chambers, and millimeter wave passive devices and sensors. 


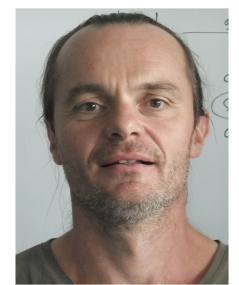

Stéphane Méric (M'08) simultaneously gratuated in 1991 from the National Institute for the Applied Sciences (INSA, Rennes, France) with an electrical engineer diploma and from the University of Rennes 1 , with a M.S. degree in "signal processing and telecommunications". He received Ph.D. (1996) in "electronics" from INSA and HDR (habilitation à diriger des recherches) from University of Rennes 1 in 2016. Since 2000, he is assistant professor at INSA and in 2005, he joined the SAPHIR team (IETR - CNRS UMR 6164, Rennes). He was interested in using SAR data in radargrammetric applications. Furthermore, he is currently working on radar system (CW, FMCW) dedicated to specific SAR applications (radar imaging in motorway context, remote sensing, MIMO configuration, passive radar imaging) and remote sensing applications. His education activities are about analog electronics, signal processing, radar and radar imaging, electromagnetic diffraction. At this time, he is the head master of the "Communication system and network" department at INSA Rennes. Dr. Stéphane Méric is co-author of more than 40 conference papers, 11 journal papers, 2 book chapters and 1 patent. He has been supervising 8 $\mathrm{PhD}$ students and currently $6 \mathrm{PhD}$ students. He was the EuRAD 2019 and the Automotive forum 2019 TPC co-chair. He has set with educational team a teaching department in an engineering school at Oujda, Morrocco.

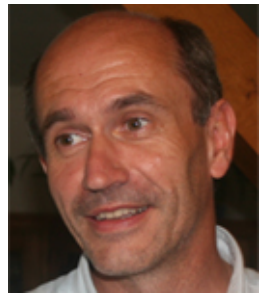

Philippe Besnier (M'04, SM'10) received the diplôme d'ingénieur degree from école universitaire d'ingénieurs de Lillle (EUDIL), Lille, France, in 1990 and the $\mathrm{Ph}$. D. degree in electronics from the university of Lille in 1993. Following a one-year period at ONERA, Meudon as an assistant scientist in the EMC division, he was with the laboratory of radio-propagation and electronics (LRPE), University of Lille, as a researcher (chargé de recherche) at the Centre National de la Recherche Scientifique (C.N.R.S.) from 1994 to 1997. From 1997 to 2002, Philippe Besnier was the director of Centre d'Etudes et de Recherches en Protection Electromagnétique (CERPEM): a non-for-profit organization for research, expertise and training in EMC and related activities, based in Laval, France. He also co-founded TEKCEM in 1998, a small business company specialized in turn-key systems for EMC measurements. Back to CNRS in 2002, he has been since then with the Institut d'Electronique et des Technologies du numéRique (IETR), Rennes, France. Philippe Besnier was appointed as CNRS senior researcher (directeur de recherche au CNRS) in 2013. He was co-head of the "antennas and microwave devices" research department of the IETR between 2012 and 2016. He headed the WAVES (electromagnetic waves in complex media) team during the first semester of 2017. Since July 2017, he is deputy director of the IETR. His research activities deal with interference analysis on cable harnesses (including electromagnetic topology), theory and application of reverberation chambers, shielding and absorbing techniques, near-field probing and uncertainty quantification in EMC modeling.

\section{Jérôme Sol}

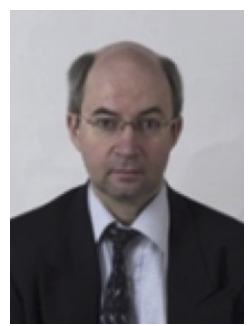

Philippe Pouliguen received the M.S. degree in signal processing and telecommunications, the Doctoral degree in electronic and the "Habilitation à Diriger des Recherches" degree from the University of Rennes 1, France, in 1986, 1990 and 2000. In 1990, he joined the Direction Générale de l'Armement (DGA) at the Centre d'Electronique de l'Armement (CELAR), now DGA Information Superiority (DGA/IS), in Bruz, France, where he was a "DGA senior expert" in electromagnetic radiation and radar signatures. He was also in charge of the EMC (Expertise and ElectoMagnetism Computation) laboratory of DGA/IS. From 2009 to 2018, Dr. Pouliguen was the head of "acoustic and radio-electric waves" scientific domain at DGA, Paris, France. Since 2018 he is Innovation Manager of the "acoustic and radio-electric waves" domain at the Agence Innovation Défense (AID). His research interests include electromagnetic scattering and diffraction, Radar Cross Section (RCS) measurement and modeling, asymptotic high frequency methods, radar signal processing and analysis, antenna scattering problems and Electronic Band Gap Materials. 